Revista de Matemática: Teoría y Aplicaciones 2002 9(1) : 11-23

CIMPA - UCR - CCSS ISSN: 1409-2433

\title{
ON THE STABILITY OF A CLASS OF POLYTOPES OF THIRD ORDER SQUARE MATRICES AND STABILITY RADIUS
}

\author{
EFRÉN VÁZquez Silva*
}

Recibido/Received: 27 Julio/July 2000

\begin{abstract}
In this work we investigate the stability properties of a convex symmetric timeinvariant third order matrix's polytope depending on a real positive parameter $r$. We apply the obtained results to the calculation of the real stability radius of a third order matrix under a certain class of affine perturbations.
\end{abstract}

Keywords: Polytope, stability radius, matrix analysis, Hurwitz stability.

\section{Resumen}

En este trabajo estudiamos las propiedads de estabilidad de un politopo simétrico y convexo de matrices de tercer orden invariantes en el tiempo, este politopo depende de un parámetro real positivo $r$. Los resultados que se obtienen son aplicados al cálculo de radio real de estabilidad, no dependiente del tiempo, de una matriz cuadrada de tercer orden sometida a cierta multiperturbación afin.

Palabras clave: Politopo, radio de estabilidad, análisis matricial, estabilidad según Hurwitz.

Mathematics Subject Classification: 34K20, 34K25.

\section{Introduction}

In Control Theory robustness plays a very important role. This property is a determining factor in the behaviour of plants and their aggregates. Because robustness takes into

${ }^{*}$ Faculty of Informatics and Mathematics, University of Holguín, Cuba. Personal address: GP 57, Holguín, CP 80100, Cuba. E-Mail: evazquez@uho.hlg.edu.cu. 
account the uncertainities that appear in the process of mathematical modelling phenomena of any type, it ensures efficiency in the construction and introduction of the flexible systems of production. Besides, a basic problem of robustness analysis is to determine to which extent the stability of a given stable system is preserved under various types of parameter perturbations.

The work [Nic98] analyzes the absolute stability problem of an automatic control system of the form:

$$
\Sigma_{u}: \quad \dot{x}=A_{0} x+b u(t),
$$

where $A_{0}=\left(a_{i j}^{0}\right) \in \mathbb{R}^{3 \times 3}$ is a constant matrix, $b \in \mathbb{R}^{3 \times 1}$, $u$ represents the control and has the form:

$$
u=c^{T} x, \quad c^{T}=\left(c_{1}, c_{2}, c_{3}\right) .
$$

Such systems may be encountered in Engineering of Control, where they serve as nominal models in plants which are characterized by flexibility of their elements, such as cranes and robots.

If information about the vector $c \in \mathbb{R}^{3 \times 1}$ is not complete, only the coordinates of this vector are known:

$$
c_{i}=c_{i}^{0}+v_{i}, \quad i=\overline{1,3}
$$

$c^{0}$ is a constant vector, while the vector $v$ belongs to the polyhedron

$$
V=\left\{v \in \mathbb{R}^{3} /\left|v_{i}\right| \leq v_{i}^{0}, i=\overline{1,3}\right\}
$$

$v$ represents the control error, the values $v_{i}^{0}$ are given or they are experimental data. Afterward substitute (I.2) in (I.1) and put the result of this operation in the system $\Sigma_{u}$, we obtain:

$$
\Sigma_{v}: \quad \dot{x}=\left(A+b v^{T}\right) x,
$$

where $A=\left(a_{i j}\right), \quad a_{i j}=a_{i j}^{0}+b_{i} c_{j}^{0} ; \quad i, j=\overline{1,3}$.

It is supposed that the system $\Sigma_{v}$ is controllable, in other words, the pair $(A, b)$ satisfies that $\operatorname{det}\left[b, A b, A^{2} b\right] \neq 0$, and two possibilities are considered:

1. That the perturbations $v$ be time-invariant. In this case the stability of the system $\Sigma_{v}$ is equivalent to the stability of interval matrices of the form:

$$
\mathrm{A}=\left[a_{i j}+b_{i} v_{j}\right]_{i, j=\overline{1,3}}, \quad v_{j} \in\left[-v_{j}^{0}, v_{j}^{0}\right] .
$$

The elements of the $\mathrm{j}$-th column of $\mathrm{A}$ are related by the parameter $v_{j}$ which varies in a symmetric interval.

2. That the perturbations $v$ be time-varying, then the stability of the system $\Sigma_{v}$ is equivalent to the stability of the differential inclusion (see [But85]):

$$
\dot{x} \in F(x)=\left\{\left(A+b v^{T}\right) x, \quad v \in V\right\} .
$$

In [Nic98] the stability properties of the system $\Sigma_{v}$ are given by the next 
Theorem 1.1 The set of matrices $A$ is asymptotically stable if and only if:

$$
\begin{aligned}
& \text { i) }\left|b_{1}\right| v_{1}^{0}+\left|b_{2}\right| v_{2}^{0}+\left|b_{3}\right| v_{3}^{0}<-t r A ; \\
& \text { ii) }\left|\operatorname{det}\left[\begin{array}{lll}
b & a_{2} & a_{3}
\end{array}\right]\right| v_{1}^{0}+\left|\operatorname{det}\left[\begin{array}{lll}
a_{1} & b & a_{3}
\end{array}\right]\right| v_{2}^{0}+\left|\operatorname{det}\left[\begin{array}{lll}
a_{1} & a_{2} & b
\end{array}\right]\right| v_{3}^{0}<-\operatorname{det} A ; \\
& \text { iii) } d^{T} v+\frac{1}{2} v^{T} C v>(\operatorname{tr} A) \sum_{p=1}^{3}(A)_{p p}-\operatorname{det} A,
\end{aligned}
$$

for the following finite set of values:

For $i \in\{1,2,3\}$ and $\vartheta_{i} \in\{-1,1\}, v_{i}=\vartheta_{i} v_{i}^{0},\{i, j, k\}=\{1,2,3\}$

a) If $c_{j j} \geq 0, c_{k k} \geq 0, c_{j j} c_{k k}-c_{k j} c_{j k}>0$

$$
-v_{1}^{0} \leq \widetilde{v}_{1}=-\frac{\left|\begin{array}{cc}
c_{i l} v_{i}+d_{l} & c_{m l} \\
c_{i m} v_{i}+d_{m} & c_{m m}
\end{array}\right|}{\left|\begin{array}{cc}
c_{j j} & c_{j k} \\
c_{k j} & c_{k k}
\end{array}\right|} \leq v_{1}^{0}, \quad\{m, l\}=\{j, k\}, \quad m \neq l,
$$

then $v_{j}=\widetilde{v}_{j}$ and $v_{k}=\widetilde{v}_{k}$.

b) In other cases the third inequality must be satisfied for the following values:

For $r \in\{j, k\}, \vartheta_{r} \in\{-1,1\}, v_{r}=\vartheta_{r} v_{r}^{0},\{r, s\}=\{j, k\}$

$\left.b^{\prime}\right)$ If $c_{s s}>0$ and $-v_{s}^{0} \leq{\widetilde{v_{s}}}_{s}=-\frac{d_{s}+c_{i s} v_{i}+c_{r s} v_{r}}{c_{s s}} \leq v_{s}^{0}$, then $v_{s}=\widetilde{v}_{s}$.

b") Otherwise $v_{s}= \pm v_{s}^{0}$.

$a_{i}, i=1,2,3$, represents the $\mathrm{i}$-th column of the matrix $A$. Here and in the sequel by $(A)_{i j}, i, j=1,2,3$ will be denoted the $i, j$-minor of the matrix $A$. In iii) the elements of the matrix $C$ and the vector $d$ are combinations of the elements of the matrix $A$ and the coordinates of the vector $b$.

The previous theorem is a necessary condition too for the stability of the differential inclusion $\dot{x}=F(x)$, with the set $F(x)$ as above.

In the current note our aim is to derive the stability properties of a class of families of third order time-invariant matrices that take their values in a convex and symmetric polytope which in turn depends on a real positive parameter whose variation represents an expansion or a contraction of the polytope.

The stability properties of these families are given in terms of the mentioned parameter, and the analyzed polytope is an extension of the third order matrix intervals considered in [Nic98] (stationary case).

The results obtained are applied to the calculation of the real stability radius of a Hurwitzstable matrix $A \in \mathbb{R}^{3 \times 3}$ under affine time-invariant multiple perturbations of the form $A \rightarrow A+\sum_{i=1}^{4} \delta_{i} B_{i}, A, B_{i} \in \mathbb{R}^{3 \times 3}, \delta_{i} \in \mathbb{R}, i=\overline{1,4}$. Moreover, we compare our new method with the existing possibilities for the calculation of the real stability radius of the matrix $A \in \mathbb{R}^{3 \times 3}$ under structured perturbation. As quantitative indicator of robustness stability 
radius has been introduced in [VanL85], [HinPri86], [HinPri86a] and it is a measure of how large may be the perturbations that conserve the stability of the system.

The paper is organized as follows: In the next section we formulate the problem and define the number $r^{*}\left(A,\left(B_{i}\right)_{i=\overline{1,4}}\right)$ that plays an important role to reach our purpose. In the Section 3 we calculate the above mentioned number and lastly (Section 4 ) we compare the obtained results with other tool of robustness analysis.

\section{Formulation of the problem}

Let $A \in \mathbb{R}^{3 \times 3}$ be a Hurwitz-estable matrix, i.e. the spectrum $\sigma(A)$ of the matrix $A$ belongs to the set $\mathbb{C}_{-}=\{\lambda \in \mathbb{C}: \Re(\lambda)<0\}$. Let $B_{i} \in \mathbb{R}^{3 \times 3}, i=\overline{1,4}$, be not all null-matrices and have the form $B_{i}=b\left(v^{(i)}\right)^{T}, i=\overline{1,4}$, where $b \in \mathbb{R}^{3 \times 1}$ is a constant vector, and the vectors $v^{(i)} \in \mathbb{R}^{3 \times 1}, i=\overline{1,4}$, have the coordinates:

$$
\begin{aligned}
& v^{(1)}=\left(v_{1}^{0}, v_{2}^{0}, v_{3}^{0}\right) \\
& v^{(2)}=\left(-v_{1}^{0}, v_{2}^{0}, v_{3}^{0}\right) \\
& v^{(3)}=\left(v_{1}^{0},-v_{2}^{0}, v_{3}^{0}\right) \\
& v^{(4)}=\left(v_{1}^{0}, v_{2}^{0},-v_{3}^{0}\right) .
\end{aligned}
$$

$v_{q}^{0}, q=1,2,3$, are given.

Obviously $\operatorname{rk}\left(B_{i}\right)=1, i=\overline{1,4}$ ( by $\operatorname{rk}(M)$ we denote the rank of the matrix $M$ ).

Let us to consider for each number $r>0$ the convex and symmetric polytope depending on parameter $r$ and formed by time-invariant matrices:

$$
\aleph\left(A,\left(B_{i}\right)_{i=\overline{1,4}}, r\right)=\operatorname{conv}\left\{A \pm r B_{i}, i=\overline{1,4}\right\} .
$$

For $\aleph\left(A,\left(B_{i}\right)_{i=\overline{1,4}}, r\right)$ we formulate the following problem: Find the values of $r>0$ such that the convex and symmetric polytope $\aleph\left(A,\left(B_{i}\right)_{i=\overline{1,4}}, r\right)$ be estable, that is to say, that each matrix $M \in \aleph\left(A,\left(B_{i}\right)_{i=\overline{1,4}}, r\right)$ be estable.

We denote by:

$$
r^{*}\left(A,\left(B_{i}\right)_{i=\overline{1,4}}\right)=\inf \left\{r>0: \aleph\left(A,\left(B_{i}\right)_{i=\overline{1,4}}, r\right) \text { contents at least one unstable matrix }\right\} .
$$

If we determine the number $r^{*}\left(A,\left(B_{i}\right)_{i=\overline{1,4}}\right)$, the stated problem will be solved because the family $\aleph\left(A,\left(B_{i}\right)_{i=\overline{1,4}}, r\right)$ is asymptotically stable iff $r<r^{*}\left(A,\left(B_{i}\right)_{i=\overline{1,4}}\right)$.

\section{Calculation of the number $r^{*}\left(A,\left(B_{i}\right)_{i=\overline{1,4}}\right)$}

In this section we study the stability of the family $\aleph\left(A,\left(B_{i}\right)_{i=\overline{1,4}}, r\right)$, for this purpose we use the well known Routh-Hurwitz criterion (see [Lan69]).

First we introduce the following definitions.

Definition 3.1 The polytope $\aleph\left(A,\left(B_{i}\right)_{i=\overline{1,4}}, r\right)$ is stable if and only if all matrices $M \in \aleph\left(A,\left(B_{i}\right)_{i=\overline{1,4}}, r\right)$ are Hurwitz-stables. 
Definition 3.2 The polytope $\aleph\left(A,\left(B_{i}\right)_{i=\overline{1,4}}, r\right)$ is unstable if there exists at least one matrix $M \in \aleph\left(A,\left(B_{i}\right)_{i=\overline{1,4}}, r\right)$ unstable.

With the matrices $A$ and $B_{i}, \quad i=\overline{1,4}$ we form new matrices depending on real parameter $r$ :

$$
M_{2 i}(r)=A-r B_{i}, \quad M_{2 i-1}(r)=A+r B_{i}, \quad i=\overline{1,4},
$$

and with their, in turns, we form a new family

$$
M(r)=\operatorname{conv}\left\{M_{j}(r), \quad j=\overline{1,8}\right\} .
$$

Evidently $\aleph\left(A,\left(B_{i}\right)_{i=\overline{1,4}}, r\right)=M(r)$ then, by the Routh-Hurwitz criterion, the considered polytope is stable iff the following inequalities hold:

$$
\begin{aligned}
\operatorname{tr} M(r) & <0 \\
\operatorname{det} M(r) & <0 \\
\operatorname{det} M(r)-(\operatorname{tr} M(r)) \sum_{p}(M(r))_{p p} & >0 .
\end{aligned}
$$

Now we introduce the following notations:

$$
\begin{aligned}
\xi^{T}= & \left(b_{1} v_{1}^{0}, b_{2} v_{2}^{0}, b_{3} v_{3}^{0}\right), \\
\eta^{T}= & \left(\left[b_{1}\left(a_{22}+a_{33}\right)-b_{2} a_{12}-b_{3} a_{13}\right] v_{1}^{0},\left[-b_{1} a_{21}+b_{2}\left(a_{11}+a_{33}\right)-b_{3} a_{23}\right] v_{2}^{0},\right. \\
& {\left.\left[-b_{1} a_{31}-b_{2} a_{32}+b_{3}\left(a_{11}+a_{22}\right)\right] v_{3}^{0}\right), } \\
\rho^{T}= & \left(\left|\begin{array}{lll}
b_{1} & a_{12} & a_{13} \\
b_{2} & a_{22} & a_{23} \\
b_{3} & a_{32} & a_{33}
\end{array}\right| v_{1}^{0},\left|\begin{array}{lll}
a_{11} & b_{1} & a_{13} \\
a_{21} & b_{2} & a_{23} \\
a_{31} & b_{3} & a_{33}
\end{array}\right| v_{2}^{0},\left|\begin{array}{lll}
a_{11} & a_{12} & b_{1} \\
a_{21} & a_{22} & b_{2} \\
a_{31} & a_{32} & b_{3}
\end{array}\right| v_{3}^{0}\right), \\
\chi^{T}= & \rho^{T}-(\operatorname{tr} A) \eta^{T}-\sum_{p=1}^{3}(A)_{p p} \xi^{T},
\end{aligned}
$$

and the transformation

$$
\beta=G \gamma, \quad G=\left(\begin{array}{cccccccc}
1 & -1 & -1 & 1 & 1 & -1 & 1 & -1 \\
1 & -1 & 1 & -1 & -1 & 1 & 1 & -1 \\
1 & -1 & 1 & -1 & 1 & -1 & -1 & 1
\end{array}\right)
$$

where $\beta \in \mathbb{R}^{3 \times 1}, G \in \mathbb{R}^{3 \times 8}$ and $\gamma \in \mathbb{R}^{8 \times 1}$.

At this point, considering the inequalities (2), and using the notations (3), we can formulate the next theorem for the stability of the polytope $M(r)$.

Theorem 3.3 Let $A \in \mathbb{R}^{3 \times 3}$ be a Hurwitz-stable matrix, let be $B_{i} \in \mathbb{R}^{3 \times 3}$, $i=\overline{1,4}$, the matrices defined in Section 2. Then the time-invariant polytope $M(r)$ is stable if and only if

$$
r<\min \left\{\pi_{1}\left(A,\left(B_{i}\right)_{i=\overline{1,4}}\right), \pi_{2}\left(A,\left(B_{i}\right)_{i=\overline{1,4}}\right), \pi_{3}\left(A,\left(B_{i}\right)_{i=\overline{1,4}}\right)\right\}=r^{*}\left(A,\left(B_{i}\right)_{i=\overline{1,4}}\right),
$$


where the numbers $\pi_{1}\left(A,\left(B_{i}\right)_{i=\overline{1,4}}\right), \pi_{2}\left(A,\left(B_{i}\right)_{i=\overline{1,4}}\right), \pi_{3}\left(A,\left(B_{i}\right)_{i=\overline{1,4}}\right)$ are the solutions of the following optimization problems
(i) $\left\{\begin{array}{l}\sum_{j=1}^{8} \gamma_{j} \longrightarrow \min \\ \operatorname{tr} A+\xi^{T} \beta=0 \\ \gamma_{j} \geq 0, \quad j=\overline{1,8}\end{array}\right.$
$(i i)\left\{\begin{array}{l}\sum_{j=1}^{8} \gamma_{j} \longrightarrow \min \\ \operatorname{det} A+\rho^{T} \beta=0 \\ \gamma_{j} \geq 0, \quad j=\overline{1,8}\end{array}\right.$
$($ iii $)\left\{\begin{array}{l}\sum_{j=1}^{8} \gamma_{j} \longrightarrow \min \\ g+\langle\chi, \beta\rangle-\beta^{T} \eta \xi^{T} \beta=0 \\ \gamma_{j} \geq 0, \quad j=\overline{1,8}\end{array}\right.$

respectively, $g=\operatorname{det} A-\operatorname{tr} A \sum_{p=1}^{3}(A)_{p p}$.

Proof: If we apply inequalities (2) to the considerd polytope, it is obtained that $M(r)$ is stable for all $r>0$ such that the inequalities $\operatorname{tr} A+\xi^{T} \beta<0$, det $A+\rho^{T} \beta<0$ and $g+\langle\chi, \beta\rangle-\beta^{T} \eta \xi^{T} \beta>0$ hold. So the theorem's statement is a direct consecuence of the Definition 3.2 and the continuity of the LHS in the inequalities (2) like functions of the parameter $r$. Finally, the equality in the expression (4) is due to Definitions 3.1, 3.2 and definition of the number $r^{*}\left(A,\left(B_{i}\right)_{i=\overline{1,4}}\right)$.

Remark 3.4 The proved Theorem 3.3 is a necessary and sufficient condition for the stability of politope $M(r)$. The theorem is a necessary condition too for the stability of the corresponding time-varying polytope $(M(r))(t)$. The polytope $M(r)$ contains the set $A$ when $r=1$, then if $r^{*}\left(A,\left(B_{i}\right)_{i=\overline{1,4}}\right)>1$ the corresponding system $\Sigma_{v}$ will be asymptotically stable (in the case when the perturbation $v$ is time-invariant).

In the two lemmas that we prove bellow, we calculate the numbers $\pi_{1}\left(A,\left(B_{i}\right)_{i=\overline{1,4}}\right)$, $\pi_{2}\left(A,\left(B_{i}\right)_{i=\overline{1,4}}\right)$ and $\pi_{3}\left(A,\left(B_{i}\right)_{i=\overline{1,4}}\right)$, which let us to obtain the number $r^{*}\left(A,\left(B_{i}\right)_{i=\overline{1,4}}\right)$.

Lemma 3.5 Let $A \in \mathbb{R}^{3 \times 3}$ and $B_{i} \in \mathbb{R}^{3 \times 3}, i=\overline{1,4}$, be matrices that satisfice the conditions of the Theorem 3.3, then:

$$
\begin{gathered}
\pi_{1}\left(A,\left(B_{i}\right)_{i=\overline{1,4}}\right)=\frac{-t r A}{\left|b_{1}\right| v_{1}^{0}+\left|b_{2}\right| v_{2}^{0}+\left|b_{3}\right| v_{3}^{0}}, \\
\pi_{2}\left(A,\left(B_{i}\right)_{i=1,4}\right)=\frac{-\operatorname{det} A}{\left|\omega_{1}\right| v_{1}^{0}+\left|\omega_{2}\right| v_{2}^{0}+\left|\omega_{3}\right| v_{3}^{0}}, \\
\text { where } w_{1}=\left|\begin{array}{lll}
b_{1} & a_{12} & a_{13} \\
b_{2} & a_{22} & a_{23} \\
b_{3} & a_{32} & a_{33}
\end{array}\right|, w_{2}=\left|\begin{array}{lll}
a_{11} & b_{1} & a_{13} \\
a_{21} & b_{2} & a_{23} \\
a_{31} & b_{3} & a_{33}
\end{array}\right|, w_{3}=\left|\begin{array}{lll}
a_{11} & a_{12} & b_{1} \\
a_{21} & a_{22} & b_{2} \\
a_{31} & a_{32} & b_{3}
\end{array}\right| .
\end{gathered}
$$

Proof: (i), (ii) are linear programming problems, so their solutions are reached in the vertexs $A \pm r B_{i}, \quad i=\overline{1,4}$ of the polytope $M(r)$. That is equivalent to looking for the smallest positive root of the algebraic equations:

$$
\operatorname{tr} A \pm r\left\langle b, v^{(i)}\right\rangle=0, \quad \operatorname{det} A \pm r\left\langle w, v^{(i)}\right\rangle=0, \quad i=\overline{1,4},
$$

where $\omega=\left(\omega_{1}, \omega_{2}, \omega_{3}\right)$, that is to say

$$
\pi_{1}\left(A,\left(B_{i}\right)_{i=\overline{1,4}}\right)=\inf \left\{r>0 / \operatorname{tr} A+r\left|\left\langle b, v^{(i)}\right\rangle\right|=0 \text { for some } i \in\{1,2,3,4\}\right\},
$$




$$
\pi_{2}\left(A,\left(B_{i}\right)_{i=\overline{1,4}}\right)=\inf \left\{r>0 / \operatorname{det} A+r\left|\left\langle w, v^{(i)}\right\rangle\right|=0 \text { for some } i \in\{1,2,3,4\}\right\}
$$

and the infimum of these sets is reached for the greatest values of $\left|\left\langle b, v^{(i)}\right\rangle\right|$ and $\left|\left\langle w, v^{(i)}\right\rangle\right|$ respectively, this imply directly the statement of the lemma.

Lemma 3.6 Let $A \in \mathbb{R}^{3 \times 3}$ and $B_{i} \in \mathbb{R}^{3 \times 3}, i=\overline{1,4}$, be matrices that satisfice the conditions of the Theorem 3.3, then $\pi_{3}\left(A,\left(B_{i}\right)_{i=\overline{1,4}}\right)=k^{*} U$, where $U$ is the solution of the following linear programming problem:

$$
\begin{array}{lll}
\sum_{j=1}^{8} u_{j} \longrightarrow \min & \\
u_{1}+\frac{\left(\xi_{1}+\xi_{2}+\xi_{3}\right)}{16\|\xi\|^{2}} \geq 0 & u_{2}-\frac{\left(\xi_{1}+\xi_{2}+\xi_{3}\right)}{16\|\xi\|^{2}} \geq 0 & u_{3}+\frac{\left(-\xi_{1}+\xi_{2}+\xi_{3}\right)}{16\|\xi\|^{2}} \geq 0 \\
u_{4}-\frac{\left(-\xi_{1}+\xi_{2}+\xi_{3}\right)}{16\|\xi\|^{2}} \geq 0 & u_{5}+\frac{\left(\xi_{1}-\xi_{2}+\xi_{3}\right)}{16\|\xi\|^{2}} \geq 0 & u_{6}-\frac{\left(\xi_{1}-\xi_{2}+\xi_{3}\right)}{16\|\xi\|^{2}} \geq 0 \\
u_{7}+\frac{\left(\xi_{1}+\xi_{2}-\xi_{3}\right)}{16\|\xi\|^{2}} \geq 0 & u_{8}-\frac{\left(\xi_{1}+\xi_{2}-\xi_{3}\right)}{16\|\xi\|^{2}} \geq 0 & \\
\left(\xi_{1}+\xi_{2}+\xi_{3}\right)\left(u_{1}-u_{2}\right)+\left(-\xi_{1}+\xi_{2}+\xi_{3}\right)\left(u_{3}-u_{4}\right)+\left(\xi_{1}-\xi_{2}+\xi_{3}\right)\left(u_{5}-u_{6}\right)+ \\
+\left(\xi_{1}+\xi_{2}-\xi_{3}\right)\left(u_{7}-u_{8}\right)=0
\end{array}
$$

and $k^{*}$ is the root of smallest absolute value of the equation $\Phi(k)=0$,

$$
\begin{aligned}
& \Phi(k):=-\frac{\xi^{T} \eta}{4\|\xi\|^{2}} k^{2}+\frac{\chi^{T} \xi}{2\|\xi\|^{2}} k+g-\left[\left(\eta_{1}+\eta_{2}+\eta_{3}\right)\left(u_{1}-u_{2}\right)+\left(-\eta_{1}+\eta_{2}+\eta_{3}\right)\left(u_{3}-u_{4}\right)\right. \\
& \left.+\left(\eta_{1}-\eta_{2}+\eta_{3}\right)\left(u_{5}-u_{6}\right)+\left(\eta_{1}+\eta_{2}-\eta_{3}\right)\left(u_{7}-u_{8}\right)\right] \frac{|k| k}{2}+\left[\left(\chi_{1}+\chi_{2}+\chi_{3}\right)\left(u_{1}-u_{2}\right)+\right. \\
& \left.+\left(-\chi_{1}+\chi_{2}+\chi_{3}\right)\left(u_{3}-u_{4}\right)+\left(\chi_{1}-\chi_{2}+\chi_{3}\right)\left(u_{5}-u_{6}\right)+\left(\chi_{1}+\chi_{2}-\chi_{3}\right)\left(u_{7}-u_{8}\right)\right]|k|,
\end{aligned}
$$

$\xi_{i}, \quad \eta_{i}, \quad \chi_{i}, i=1,2,3$, are the coordinates of the vectors $\xi^{T}, \eta^{T}, \chi^{T}$ respectively.

Proof: Let us to apply the Lagrange's method (see, for example [GalTij91]) in order to solve the problem iii).

The corresponding Lagrange's function has the form:

$$
\mathrm{L}(\gamma, \lambda)=\lambda_{0} f_{0}\left(\gamma_{1}, \ldots, \gamma_{8}\right)-\sum_{j=1}^{8} \lambda_{j} f_{j}\left(\gamma_{1}, \ldots, \gamma_{8}\right)+\lambda_{9} f_{9}\left(\gamma_{1}, \ldots, \gamma_{8}\right)
$$

where

$$
\begin{gathered}
f_{0}\left(\gamma_{1}, \ldots, \gamma_{8}\right)=\sum_{j=1}^{8} \gamma_{j}, f_{j}\left(\gamma_{1}, \ldots, \gamma_{8}\right)=\gamma_{j}, j=\overline{1,8} \\
f_{9}\left(\gamma_{1}, \ldots, \gamma_{8}\right)=g+\left\langle G^{T} \chi, \gamma\right\rangle-\gamma^{T} G^{T} \eta \xi^{T} G \gamma
\end{gathered}
$$

Apllying the necessary extreme condition to the function $\mathrm{L}(\gamma, \lambda)$ given in (5), we obtain the following algebraic equations system:

$$
\lambda_{0}\left(\begin{array}{c}
1 \\
\vdots \\
1
\end{array}\right)-\left(\begin{array}{c}
\lambda_{1} \\
\vdots \\
\lambda_{8}
\end{array}\right)+\lambda_{9}\left[G^{T} \chi-2 G^{T} \eta \xi^{T} G \gamma\right]=0
$$


In the system (6) we consider $\lambda_{9} \neq 0$ (in other case the problem lacks of interest) and we write it in the form:

$$
2 G^{T} \eta \xi^{T} G \gamma=G^{T} \chi+\Lambda
$$

where

$$
\Lambda=\frac{1}{\lambda_{9}}\left[\lambda_{0}\left(\begin{array}{c}
1 \\
\vdots \\
1
\end{array}\right)-\left(\begin{array}{c}
\lambda_{1} \\
\vdots \\
\lambda_{8}
\end{array}\right)\right] \in \mathbb{R}^{8}
$$

The system (7) has solution when $\Lambda \in \operatorname{Im}\left(G^{T}\right)$, i.e. $\Lambda=G^{T} h, h \in \mathbb{R}^{3}$. We can rewrite the system (7) as follows:

$$
2 G^{T} \eta \xi^{T} G \gamma=G^{T}(\chi+h)
$$

¿From the last equation we have that:

$$
G^{T}\left(2 \eta \xi^{T} G \gamma-(\chi+h)\right)=0
$$

and we obtain directly

$$
2 \eta \xi^{T} G \gamma=(\chi+h)
$$

by virtue of injectivity of the linear application $G^{T}: \mathbb{R}^{3} \rightarrow \mathbb{R}^{8}$. So, every solution of the equation (8) is solution of the equation (9) too and vice versa.

Now consider the equation

$$
16 \eta \xi^{T} \beta=(\chi+h) .
$$

If $\beta$ is solution of (10), then $\gamma=G^{T} \beta$ is solution of (9) by virtue of the relationship $G G^{T}=8 I_{3}$, where $I_{3}$ is the unit matrix of third order.

Let us to look for the solution of (10). Such solution will exist if the vector $\chi+h$ is multiple of the vector $\eta: \chi+h=k \eta, k$-constant. Then, in place of (10) we have:

$$
16 \eta \xi^{T} \beta=k \eta \text {. }
$$

We look for a particular solution of the equation (11) in the form: $\beta=s \xi$. By (11) it is obtained that

$$
16 \eta \xi^{T} s \xi=k \eta \Rightarrow 16 s \xi^{T} \xi \eta=k \eta \Rightarrow s=\frac{k}{16\|\xi\|^{2}}
$$

from where $\beta=\frac{k \xi}{16\|\xi\|^{2}}$.

But we must find the general solution of the system (8). Taking into account that, by virtue of equivalence beetwen the systems (8) and (9), $G^{T} \beta$ is a particular solution of (8) and the general solution that we want has the form:

$$
\widetilde{\gamma}=\gamma+W
$$


where

$$
\gamma=G^{T} \beta=\frac{k}{16\|\xi\|^{2}}\left(\begin{array}{c}
\xi_{1}+\xi_{2}+\xi_{3} \\
-\left(\xi_{1}+\xi_{2}+\xi_{3}\right) \\
-\xi_{1}+\xi_{2}+\xi_{3} \\
-\left(-\xi_{1}+\xi_{2}+\xi_{3}\right) \\
\xi_{1}-\xi_{2}+\xi_{3} \\
-\left(\xi_{1}-\xi_{2}+\xi_{3}\right) \\
\xi_{1}+\xi_{2}-\xi_{3} \\
-\left(\xi_{1}+\xi_{2}-\xi_{3}\right)
\end{array}\right)
$$

$W \in \operatorname{ker}\left(\eta \xi^{T} G\right)$, besides

$$
\begin{aligned}
\operatorname{ker}\left(\eta \xi^{T} G\right)= & \left\{W \in \mathbb{R}^{8} /\left(\xi_{1}+\xi_{2}+\xi_{3}\right)\left(W_{1}-W_{2}\right)+\right. \\
& +\left(-\xi_{1}+\xi_{2}+\xi_{3}\right)\left(W_{3}-W_{4}\right)+ \\
& +\left(\xi_{1}-\xi_{2}+\xi_{3}\right)\left(W_{5}-W_{6}\right)+ \\
& \left.+\left(\xi_{1}+\xi_{2}-\xi_{3}\right)\left(W_{7}-W_{8}\right)=0\right\} .
\end{aligned}
$$

We have transformed the problem iii) with cuadratic restriction in a new linear programming problem. Concretely:

$$
\begin{array}{lll}
\sum_{j=1}^{8} W_{j} \longrightarrow \min & \\
W_{1}+\frac{k\left(\xi_{1}+\xi_{2}+\xi_{3}\right)}{16\|\xi\|^{2}} \geq 0 & W_{2}-\frac{k\left(\xi_{1}+\xi_{2}+\xi_{3}\right)}{16\|\xi\|^{2}} \geq 0 & W_{3}+\frac{k\left(-\xi_{1}+\xi_{2}+\xi_{3}\right)}{16\|\xi\|^{2}} \geq 0 \\
W_{4}-\frac{k\left(-\xi_{1}+\xi_{2}+\xi_{3}\right)}{16\|\xi\|^{2}} \geq 0 & W_{5}+\frac{k\left(\xi_{1}-\xi_{2}+\xi_{3}\right)}{16\|\xi\|^{2}} \geq 0 & W_{6}-\frac{k\left(\xi_{1}-\xi_{2}+\xi_{3}\right)}{16\|\xi\|^{2}} \geq 0 \\
W_{7}+\frac{k\left(\xi_{1}+\xi_{2}-\xi_{3}\right)}{16\|\xi\|^{2}} \geq 0 & W_{8}-\frac{k\left(\xi_{1}+\xi_{2}-\xi_{3}\right)}{16\|\xi\|^{2}} \geq 0 & \\
\left(\xi_{1}+\xi_{2}+\xi_{3}\right)\left(W_{1}-W_{2}\right)+\left(-\xi_{1}+\xi_{2}+\xi_{3}\right)\left(W_{3}-W_{4}\right)+\left(\xi_{1}-\xi_{2}+\xi_{3}\right)\left(W_{5}-W_{6}\right)+ \\
+\left(\xi_{1}+\xi_{2}-\xi_{3}\right)\left(W_{7}-W_{8}\right)=0
\end{array}
$$

By change of variables $\frac{W_{j}}{|k|}=u_{j}, j=\overline{1,8}$, the above problem is transformed in a new problem:

$$
\begin{array}{ll}
\sum_{j=1}^{8} u_{j} \longrightarrow \min & \\
u_{1}+\operatorname{sign}(k) \frac{\left(\xi_{1}+\xi_{2}+\xi_{3}\right)}{16\|\xi\|^{2}} \geq 0 & u_{2}-\operatorname{sign}(k) \frac{\left(\xi_{1}+\xi_{2}+\xi_{3}\right)}{16\|\xi\|^{2}} \geq 0 \\
u_{3}+\operatorname{sign}(k) \frac{\left(-\xi_{1}+\xi_{2}+\xi_{3}\right)}{16\|\xi\|^{2}} \geq 0 & u_{4}-\operatorname{sign}(k) \frac{\left(-\xi_{1}+\xi_{2}+\xi_{3}\right)}{16\|\xi\|^{2}} \geq 0 \\
u_{5}+\operatorname{sign}(k) \frac{\left(\xi_{1}-\xi_{2}+\xi_{3}\right)}{16\|\xi\|^{2}} \geq 0 & u_{6}-\operatorname{sign}(k) \frac{\left(\xi_{1}-\xi_{2}+\xi_{3}\right)}{16\|\xi\|^{2}} \geq 0 \\
u_{7}+\operatorname{sign}(k) \frac{\left(\xi_{1}+\xi_{2}-\xi_{3}\right)}{16\|\xi\|^{2}} \geq 0 & u_{8}-\operatorname{sign}(k) \frac{\left(\xi_{1}+\xi_{2}-\xi_{3}\right)}{16\|\xi\|^{2}} \geq 0
\end{array}
$$




$$
\begin{aligned}
& \left(\xi_{1}+\xi_{2}+\xi_{3}\right)\left(u_{1}-u_{2}\right)+\left(-\xi_{1}+\xi_{2}+\xi_{3}\right)\left(u_{3}-u_{4}\right)+\left(\xi_{1}-\xi_{2}+\xi_{3}\right)\left(u_{5}-u_{6}\right)+ \\
& +\left(\xi_{1}+\xi_{2}-\xi_{3}\right)\left(u_{7}-u_{8}\right)=0
\end{aligned}
$$

Here we need to consider the cases $k>0$ and $k<0$, however it is sufficient to solve one of it because the solution for $k$ coincides with the solution for $-k$. At this moment we can calculate $W_{j}=|k| u_{j}, j=\overline{1,8}$, and thus the coordenates of the vector $\widetilde{\gamma}$. The value of the parametr $k$ is obtained from the fact that $\widetilde{\gamma}$ must satisfy the restriction $f_{9}(\widetilde{\gamma})=0$ corresponding to the problem iii), that is to say, we obtain that $k$ is the root of samllest absolute value of the polynomial $\Phi(k)$, denoted by $k^{*}$.

Obviously, in accordance with definition of real time-invariant stability radius and definitions of the numbers $\pi_{1}\left(A,\left(B_{i}\right)_{i=\overline{1,4}}\right), \pi_{2}\left(A,\left(B_{i}\right)_{i=\overline{1,4}}\right)$ and $\pi_{3}\left(A,\left(B_{i}\right)_{i=\overline{1,4}}\right)$, we conclude that $r_{\mathbb{R}}\left(A,\left(B_{i}\right)_{i=\overline{1,4}}, \mathbb{C}_{+}\right)=r^{*}\left(A,\left(B_{i}\right)_{i=\overline{1,4}}\right)$.

In the next we expose in an example how to apply the results obtained.

Example 3.7

$$
\begin{gathered}
A=\left(\begin{array}{ccc}
-1 & -1 & 1 \\
3 & -1 & 3 \\
-2 & 1 & -4
\end{array}\right), \quad b=\left(\begin{array}{c}
1 \\
-1 \\
1
\end{array}\right), \quad v^{0}=\left(\begin{array}{c}
-1 \\
2 \\
1
\end{array}\right) . \\
\pi_{1}\left(A,\left(B_{i}\right)_{i=\overline{1,4}}\right)=3, \quad \pi_{2}\left(A,\left(B_{i}\right)_{i=\overline{1,4}}\right)=\frac{1}{2} .
\end{gathered}
$$

By the other hand $\xi^{T}=(-1,-2,1), \quad \eta^{T}=(7,-2,1), \quad \chi^{T}=(67,-2,1), \quad g=60$

$\widetilde{\gamma}^{T}=\left(\frac{-k}{48}+W_{1}, \frac{k}{48}+W_{2}, W_{3}, W_{4}, \frac{k}{48}+W_{5}, \frac{-k}{48}+W_{6}, \frac{-k}{48}+W_{7}, \frac{k}{48}+W_{8}\right)^{T}$

$\operatorname{ker}\left(\eta \xi^{T} G\right)=\left\{W \in \mathbb{R}^{8} /-W_{1}+W_{2}+W_{5}-W_{6}+2\left(W_{8}-W_{7}\right)=0\right\}$.

In the new variables we need to solve the following linear programming problem:

$$
\left\{\begin{array}{llll}
\sum_{j=1}^{8} u_{j} \longrightarrow \min & & \\
u_{1}-\frac{1}{48} \geq 0 & u_{2}+\frac{1}{48} \geq 0 & u_{3} \geq 0 & u_{4} \geq 0 \\
u_{5}+\frac{1}{48} \geq 0 & u_{6}-\frac{1}{48} \geq 0 & u_{7}-\frac{1}{24} \geq 0 & u_{8}+\frac{1}{24} \geq 0 \\
& & -u_{1}+u_{2}+u_{5}-u_{6}-2\left(u_{7}-u_{8}\right)=0
\end{array}\right.
$$

The solution is $U=\frac{1}{8}$, the equations for the parametr $k$ are:

$$
-\frac{1}{12} k^{2}-\frac{90}{12} k+60=0, \frac{1}{4} k^{2}-\frac{5}{6} k+60=0,
$$

so $k^{*}=7.39273$ and thus $\pi_{3}\left(A,\left(B_{i}\right)_{i=\overline{1,4}}\right)=0.924094$.

$$
\Longrightarrow r^{*}\left(A,\left(B_{i}\right)_{i=\overline{1,4}}\right)=\frac{1}{2} \text {. }
$$

the matrix of the corresponding system $\Sigma_{v}$ is stable, while the real time-invariant stability radius of the matrix system $\dot{x}=\Omega x, \Omega \in M(r)$, is $r_{\mathbb{R}}\left(A,\left(B_{i}\right)_{i=\overline{1,4}}, \mathbb{C}_{+}\right)=0.5$ 


\section{Comparison with a previous result}

In the previous section we have obtained a new method in order to calculate the real time-invariant stability radius of a matrix $A \in \mathbb{R}^{3 \times 3}$ under certain affine perturbation class (the rank of the perturbation is one). By this reason we refer an earlier method by Hinrichsen and Pritchard, obtained from results of Qiu et alter ([QBRDYD95]). (Other results closely related to results obtained by Hinrichsen and Pritchard can be found in [Doy82],[PacDoy93]).

Let us to consider the system

$$
\dot{x}=A x,
$$

where the matrix $A \in \mathbb{R}^{3 \times 3}$ is Hurwitz stable. Joining with the system (12) we consider the perturbed system

$$
\dot{x}=\left(A+b v^{T}\right) x,
$$

$b \in \mathbb{R}^{3 \times 1}$ is a constant vector, while $v \in \mathbb{R}^{3 \times 1},\left|v_{i}\right| \leq v_{i}^{(0)}, i=\overline{1,3}$, represents the uncertainity of the perturbation.

Let us to put $C=\operatorname{diag}\left(v_{1}^{(0)}, v_{2}^{(0)}, v_{3}^{(0)}\right)$ and $\Delta=\left[\delta_{1}, \delta_{2}, \delta_{3}\right]$, hence we can rewrite the perturbed system (13) in the form:

$$
\dot{x}=(A+b \Delta C) x .
$$

In the system (14) the uncertainity in the perturbation is represented in the form

$v_{i}=\delta_{i} v_{i}^{(0)},\left|\delta_{i}\right| \leq 1$ (recall that we consider the values $v_{i}^{(0)}$ known). As perturbation norm we use infinity norm such that $\|\Delta\|_{\infty} \leq 1$.

Definition 4.1 The real stability radius of the matrix $A$ with respect to time-invariant perturbations of structure $(b, C)$, is defined by:

$$
r_{\mathbb{R}}\left(A, b, C, \mathbb{C}_{+}\right)=\inf \left\{\|\Delta\|: \quad \sigma(A+b \Delta C) \cap \mathbb{C}_{+} \neq \emptyset\right\}
$$

where $\|\cdot\|$ is an arbitrary norm.

Thus, when the class of perturbations is $\boldsymbol{\Delta}=\mathbb{R}^{l \times q}$ and $l=1$ or $q=1$, it holds the following:

Proposition 4.2 ([HinPri2000])

$$
r_{\mathbb{R}}\left(A, b, C, \mathbb{C}_{+}\right)=\left[\max _{\omega \in \mathbb{R}} \operatorname{dist}(X(i \omega), \mathbb{R} Y(i \omega))\right]^{-1},
$$

where $X(s), Y(s)$ are the real and the imaginary parts of the transfer matrix $G(s)=C(s I-A)^{-1} b$ associated with the perturbed system (14). 


\subsection{Calculation of $r_{\mathbb{R}}\left(A, b, C, \mathbb{C}_{+}\right)$}

Let us apply the Proposition 4.2 to the Example 3.7.

$$
\begin{gathered}
A=\left(\begin{array}{ccc}
-1 & -1 & 1 \\
3 & -1 & 3 \\
-2 & 1 & -4
\end{array}\right), \quad b=\left(\begin{array}{c}
1 \\
-1 \\
1
\end{array}\right), \quad v^{0}=\left(\begin{array}{c}
-1 \\
2 \\
1
\end{array}\right), \quad C=\left(\begin{array}{ccc}
-1 & o & 0 \\
0 & 2 & 0 \\
0 & 0 & 1
\end{array}\right) \\
\left(\omega^{2} I+A^{2}\right)^{-1}=\frac{1}{\varphi(\omega)}\left(\begin{array}{ccc}
\omega^{4}+18 \omega^{2}-19 & -3 \omega^{2}-27 & 8 \omega^{2}-28 \\
12 \omega^{2}+48 & \omega^{4}+13 \omega^{2}+36 & 12 \omega^{2}+48 \\
-13 \omega^{2}+23 & 3 \omega^{2}+27 & \omega^{4}-3 \omega^{2}+32
\end{array}\right) \\
\varphi(\omega):=\operatorname{det}\left(\omega^{2} I+A^{2}\right)=\left(\omega^{2}+1\right)\left(\omega^{2}+4\right)\left(\omega^{2}+9\right), \quad A . b=\left(\begin{array}{c}
1 \\
7 \\
-7
\end{array}\right) .
\end{gathered}
$$

It is easy to see that:

$X(i \omega)=-C\left(\omega^{2} I+A^{2}\right)^{-1} A b, \quad Y(i \omega)=-\omega C\left(\omega^{2} I+A^{2}\right)^{-1} b$,

then

$$
\begin{gathered}
X(i \omega)=\frac{1}{\varphi(\omega)}\left(\begin{array}{c}
\omega^{4}-95 \omega^{2}-12 \\
-14 \omega^{4}-38 \omega^{2}+712 \\
7 \omega^{4}-29 \omega^{2}+12
\end{array}\right), \quad Y(i \omega)=\frac{\omega}{\varphi(\omega)}\left(\begin{array}{c}
\omega^{4}-7 \omega^{2}-20 \\
2 \omega^{4}-22 \omega^{2}-128 \\
-\omega^{4}+19 \omega^{2}-28
\end{array}\right) . \\
\quad \operatorname{dist}(X(i \omega), \mathbb{R} Y(i \omega))=\|\cdot\|_{1}=\min _{\alpha \in \mathbb{R}} \sum_{k=1}^{3}\left|X_{k}(i \omega)-\alpha Y_{k}(i \omega)\right|= \\
=\frac{1}{\varphi(\omega)} \min _{\alpha \in \mathbb{R}}\left[\left|\omega^{4}-95 \omega^{2}-12-\alpha\left(\omega^{5}-7 \omega^{3}-20 \omega\right)\right|+\mid-14 \omega^{4}-38 \omega^{2}+72-\right. \\
\left.-\alpha\left(2 \omega^{5}-22 \omega^{3}-128 \omega\right)|+| 7 \omega^{4}-29 \omega^{2}+12-\alpha\left(\omega^{5}-19 \omega^{3}+28 \omega\right) \mid\right] .
\end{gathered}
$$

By Proposition 4.2 an algorithm may be obtained in order to calculate the stability radius, but in order to solve the above extremal problem the great difficulty is related with the determination of the sign, respect to $\alpha$, of each of the polynomials on variable $\omega$. The new method that we propose is more simple than the above way, and it would help in the problem of computing the real time-invariant stability radius for the studied perturbation class with bigger rank, i.e. rank 2 and 3.

\section{References}

[But85] Butkovskii, A.G. (1985) Fazabie partreti uprabliaemij dinamicheskij sistem. Editorial Nauka. Moscú (In Russian).

[Doy82] Doyle, J.C. (1982) "Analysis of feedback systems with structured uncertainties", IEEE Proc., Part D 129: 242-250.

[HinPri86] Hinrichsen, D,; Pritchard, A.J. (1986) "Stability radii of linear systems", Systems \& Control Letters 7: 1-10. 
[HinPri86a] Hinrichsen, D.; Pritchard, A.J. (1986) "Stability radius for structured perturbations and the algebraic Riccati equation", Systems \& Control Letters 8: 105-113.

[HinPri2000] Hinrichsen, D.; Pritchard, A.J. (2000) Dynamical Systems Theory. (manuscript, February, 2000).

[Lan69] Lancaster, P. (1969) Theory of Matrices. Academic Press, Inc. New York, London.

[Nic98] Nicado, M. (1998) Estudio del Comportamiento de Sistemas de Control Automático de Tercer Orden con Perturbaciones Estacionarias y No Estacionarias. Tesis de Doctorado, Universidad Central de las Villas, Santa Clara, Cuba.

[PacDoy93] Packard, A.; Doyle, J.C. (1993) "The complex structured singular value", Automatica 29(1): 71-109.

[QBRDYD95] Qiu, L.; Bernhardsson, B.; Rantzer, A.; Davison, E.J.; Young, P.M.; Doyle, J.C. (1995) "A formula for computation of the real stability radius", Automatica 31: 879-890.

[VanL85] Van Loan, C. (1985) "How near is a stable matrix to an unstable matrix?", Contemporary Math. 47: 465-477. 